\title{
Peri-Implant Bone Loss Evaluation Around Dental Implants Placed into Alveolar Cleft Sites
}

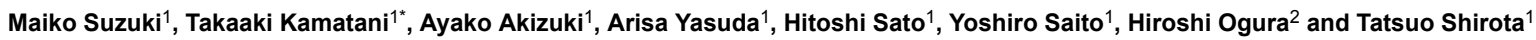 \\ ${ }^{1}$ Department of Oral and Maxillofacial Surgery, Showa University School of Dentistry, Tokyo, Japan \\ ${ }^{2}$ Department of Information Science, Showa University, Tokyo, Japan
}

"Corresponding author: Takaaki Kamatani, Department of Oral and Maxillofacial Surgery, Showa University School of Dentistry, 2-1-1 Kitasennzoku, Ota City, Tokyo 145-8515, Japan, Tel: +81 33787 1151; Fax: +81 35498 1543; E-mail: kamatani@dent.showa-u.ac.jp

Received date: Oct 15, 2016; Accepted date: Nov 01, 2016; Published date: Nov 08, 2016

Copyright: (c) 2016 Suzuki M, et al. This is an open-access article distributed under the terms of the Creative Commons Attribution License, which permits unrestricted use, distribution, and reproduction in any medium, provided the original author and source are credited.

\begin{abstract}
Objectives: This study was to analyze factors that affect peri-implant bone loss around dental implants placed into alveolar cleft sites.

Methods: Thirty-one patients with cleft lip and palate (total 46 implants) who underwent implant treatment following secondary bone graft to the alveolar cleft were included in this study. Evaluation at the peri-implant bone level was measured using standard X-ray images. Analysis of covariance was performed using the amount of periimplant bone loss as the objective variable and sex, cleft type, age at bone grafting of alveolar clefts, time since completion of implant treatment, type of implant, length of the implant, presence or absence of vestibuloplasty, and presence or absence of bone augmentation with simultaneous implant placement as the eight explanatory variables.
\end{abstract}

Results: Of the eight explanatory variables, the factors that affected the amount of peri-implant bone resorption were sex, presence or absence of vestibuloplasty, and length of the implant. The amount of peri-implant bone resorption was greater in men than in women $(p=0.004)$, and those that underwent vestibuloplasty also tended to have a greater amount of bone resorption $(p=0.002)$.

Conclusions: In implant treatment for alveolar cleft sites, a variety of factors that are not seen in non-cleft, individuals are intricately linked. Detailed analysis of factors that affect peri-implant bone loss is therefore needed in larger a subject sample in order to establish guidelines for implant treatment of alveolar cleft sites.

Keywords: Alveolar cleft; Dental implant; Marginal bone loss; Retrospective study

\section{Introduction}

Secondary bone grafting of alveolar cleft, in which permanent teeth adjacent to the cleft are encouraged to erupt or correctively displaced, is considered the most ideal method of occlusal reconstruction in cases of cleft lip and palate $[1,2]$. However, the utility of choosing implant treatment as a method that does not place a burden on the teeth adjacent to the alveolar cleft has been reported in cases where occlusal reconstruction is difficult with orthodontic treatment alone for reasons such as missing permanent teeth [3,4]. Matsui et al. [5] placed implants in bone bridges formed by secondary bone grafts to alveolar clefts and reported an implant survival rate of $98.6 \%$ in cleft lip and palate patients who underwent 5 years or more of follow-up. Meanwhile, Wang et al. [6] collected and analyzed clinical studies conducted to date and reported a $91.5 \%$ implant survival rate in alveolar cleft area over a 54.3-month follow-up period. Ferreira et al. [7] reported a 94.3\% implant survival rate in alveolar cleft area over a 34-month follow-up period.

The aforementioned reports suggest that a survival rate almost equal to that of non-cleft jaws could be obtained with implants placed in grafted alveolar cleft sites. Nonetheless, when evaluating the clinical outcomes of implants, the degree of peri-implant bone loss, in addition to the presence or absence of implant detachment, is an important evaluating factor. However, there have been no reports analyzing the changes that occur at the peri-implant alveolar bone level in the bone bridge following secondary bone grafting, so the facts are unclear. Many factors that affect changes at the peri-implant bone level have been reported, including sex, age, site of implant placement, length and diameter of the implant, surface properties of the implant, and bone quality of the jawbone $[8,9]$.

In the present study, we analyzed the factors that affect changes at the peri-implant bone level using X-ray images taken for necessary post-treatment diagnosis in patients who underwent implant treatment of the jaw following secondary bone graft at our department.

The present study was conducted with the approval of the ethics committee of Showa University School of Dentistry (2011-008).

\section{Materials and Methods}

Thirty-one patients (11 male and 20 female) with cleft lip and palate who underwent implant treatment (46 implants) following secondary bone graft to the cleft at Showa University Dental Hospital during the 15-year, 6-month period from February 1995 to August 2010 were include in this study. Evaluation at the peri-implant bone level was measured using standard X-ray images. Twenty-two cases had unilateral cleft lip and palate, and 9 cases had bilateral cleft lip and palate. 
Age at the time of cleft jawbone grafting ranged from 14 to 30 years (mean: 19.8 years), and all cases had undergone autologous cancellous iliac bone grafting to get adequate alveolar volume that was same width and height with both sides of the teeth. Orthodontic treatment was performed in these subjects while securing the implant placement site. Age at the time of implant surgery ranged from 14 to 30 years (mean: 21.4 years; Table 1). Implant placement was performed in line with recommended procedures under local anesthesia following completion of orthodontic treatment. Implant shoulder level was equal to that of the bone crest.

\begin{tabular}{|l|l|l|l|}
\hline Variable & Mean & (SD) & Range \\
\hline Age of grafted bone in alveolar cleft (year) & 19.8 & 0.7 & $14-30$ \\
\hline Age of placed implants (year) & 21.4 & 0.7 & $14-30$ \\
\hline Observation period (month) & 40.7 & 4.9 & $8-104.9$ \\
\hline Marginal bone loss (mm) & 1.5 & 0.7 & $0.8-4.1$ \\
\hline
\end{tabular}

The average age of cleft jawbone grafting was 19.8 years old. The average age of implant surgery was 21.4 years old. The average of the observation period was 40.7 months. As for the maximum of peri-implant bone loss, $4.1 \mathrm{~mm}$, the average was $1.5 \mathrm{~mm}$.

Table 1: Age of operation and peri-implant bone loss

The types of implant used were 13 Hydroxyapatite (HA)-coated implants (Integral ${ }^{\circ}$ Implant System; Calcitek, Carlsbad, CA), 10 machined surface-type titanium implants (Brånemark System MKII Implants; Nobel Biocare Holding AG, Zürich, Switzerland), and 23 rough surface-type titanium implants (Brånemark System TiU MKIII Implants; Nobel Biocare Holding AG, Zürich, Switzerland).

All implants had a diameter of $3.75 \mathrm{~mm}$. Implants in lengths of 15 $\mathrm{mm}, 13 \mathrm{~mm}$, and $11.5 \mathrm{~mm}$ were used (Table 2). In addition, 20 cases with insufficient bone quantity received a milled bone graft of cortical bone harvested from the mandibular anterior ascending ramus during the first surgery. Thirteen cases with a shallow oral vestibule and movable mucosa covering the peri-implant area underwent vestibuloplasty combined with a palatal mucosa graft during the second surgery.

\begin{tabular}{|c|c|c|c|}
\hline Type of implant & $\begin{array}{l}\text { Number of } \\
\text { case }\end{array}$ & Length(mm & $\begin{array}{l}\text { Number } \\
\text { implants }\end{array}$ \\
\hline \multirow{2}{*}{ HA coarted implant } & \multirow{2}{*}{9} & 15 & 10 \\
\hline & & 13 & 3 \\
\hline \multirow{2}{*}{$\begin{array}{l}\text { Machined surface type } \\
\text { implant }\end{array}$} & \multirow{2}{*}{8} & 15 & 6 \\
\hline & & 13 & 4 \\
\hline \multirow{3}{*}{ Rough surface type implant } & \multirow{3}{*}{14} & 15 & 5 \\
\hline & & 13 & 10 \\
\hline & & 11.5 & 8 \\
\hline Total & 31 & & 46 \\
\hline
\end{tabular}

The types of implant used were Hydroxyapatite (HA)-coated implants, machined surface-type titanium implants, and rough surface-type titanium implants. A comparatively long Implant more than $13 \mathrm{~mm}$ used in 38 of all.

Table 2: Type of implant and number of inserts implants
The same surgeon performed all these surgeries. Furthermore, maintenance was performed every 3 to 6 months after completion of treatment to examine the peri-implant gingiva, check the prosthetic devices and provide oral hygiene guidance. The follow-up period after completion of implant treatment was 8-104.9 months (mean: 46.7 months).

\section{Evaluation of peri-implant bone loss}

Implant length and maximum distance from the alveolar crest to the inferior margin of the implant collar were measured based on standard $\mathrm{X}$-ray images using $\mathrm{x}$-ray image viewer. The actual amount of bone resorption was calculated using the following formula based on the length of the embedded implant and the length of the implant measured on film (Figure 1). In addition, the same examiner took these measurements three times each at different times and used the mean as the measured value.

$$
y=a x \div b
$$

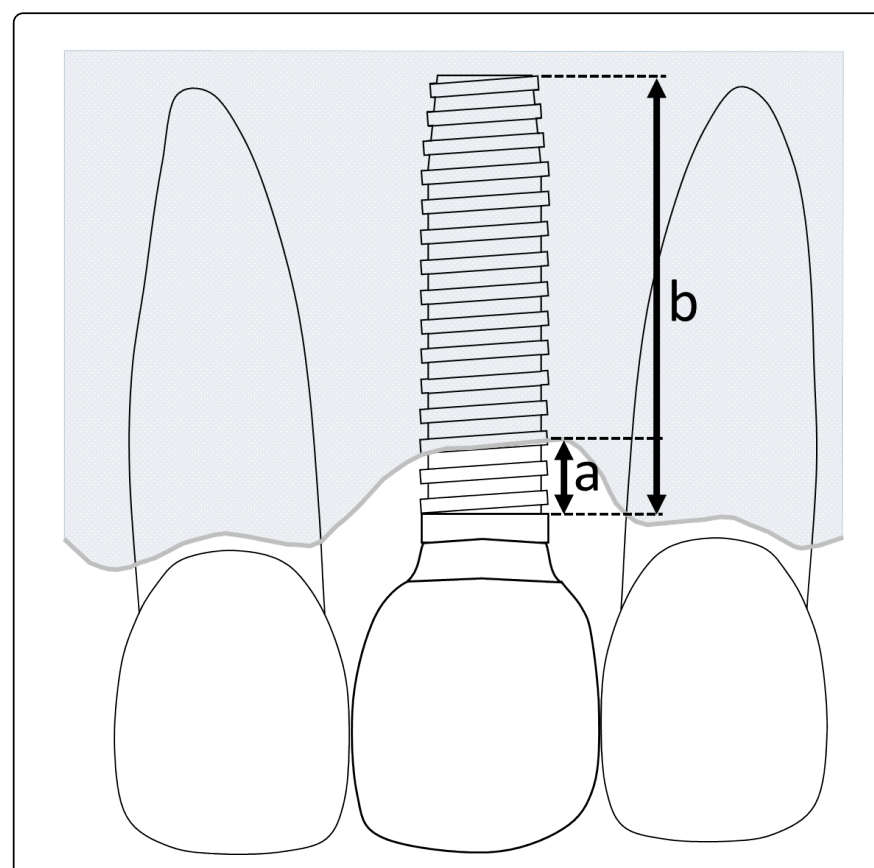

Figure 1: Calculation of amount of bone loss. Bone loss was calculated based on implant length and maximum distance from the platform to the inferior margin on X-ray images. $y=a x \div b$; $y$ : Amount of bone loss, a: Amount of bone loss on film, x: Actual implant length, b: Implant length on film

\section{Statistical analysis}

In order to examine the correlation between peri-implant bone loss and factors considered to have a potential effect on changes, an analysis of covariance with each objective variable was performed with amount of bone loss as the objective variable and sex, cleft type, age at bone grafting of alveolar clefts, follow-up period after completion of implant treatment, type of implant, length of the implant, presence or absence of vestibuloplasty, and presence or absence of bone augmentation with simultaneous implant placement as the eight explanatory variables. 
Page 3 of 5

The level of significance was set at $5 \%$. The statistical analysis was carried out in R package (Ver. 2.12.0) [10].

\section{Results}

\section{Survival of dental implants and peri-implant bone loss}

Osseointegration was achieved in all 31 cleft lip and palate patients. No implant disturbance or removal was seen. The amount of periimplant bone loss was 0.8-4.1 mm (mean: $1.5 \mathrm{~mm}$; Figure 2).

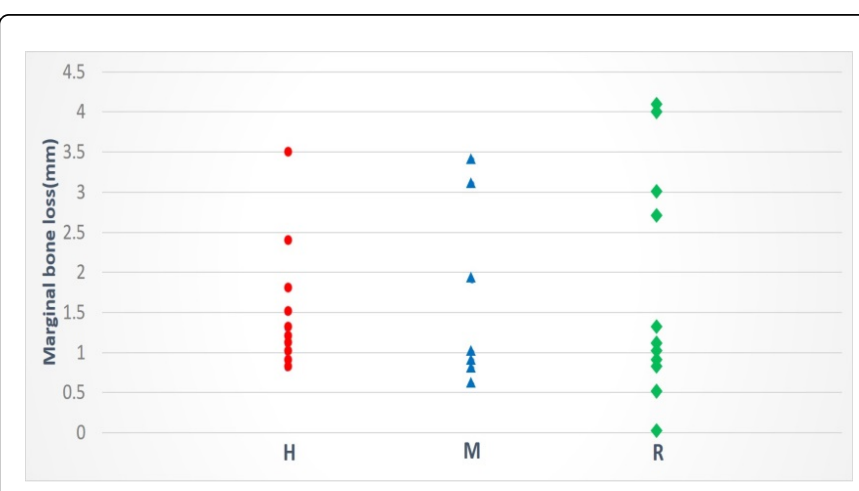

Figure 2: Effect of implant type on bone loss. No statistically significant differences were seen between type of implant and amount of peri-implant bone loss $(\mathrm{p}=0.286)$. H: Hydroxyapatitecoated implant, M: Machined surface-type titanium implant, R: Rough surface-type titanium implant.

\section{Statistical analysis}

Analysis of covariance for amount of bone loss was performed with a statistical model using all explanatory variables, i.e., sex, cleft type, age at bone grafting of alveolar clefts, follow-up period, type of implant, length of the implant, presence or absence of vestibuloplasty, and presence or absence of bone augmentation with simultaneous implant placement. Variables where no significant differences were detected were successively omitted to find the optimal combination of explanatory variables with the smallest Akaike Information Criterion (AIC) [10].

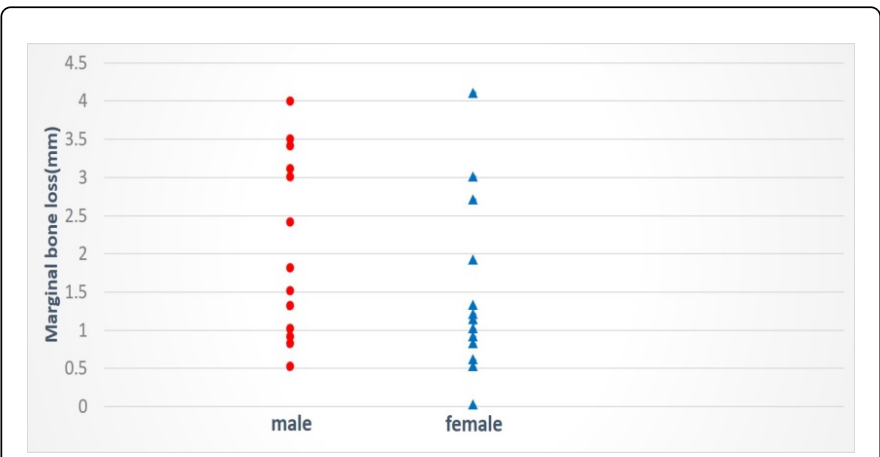

Figure 3: Effect of sex on peri-implant bone loss. Male exhibited a greater amount of bone loss than female $(\mathrm{p}=0.004)$.

As a result, a simplified statistical model using four of the initial eight explanatory variables (type of implant, length of the implant, sex and presence or absence of vestibuloplasty) was found to be the best model in an analysis of covariance (AIC=178.41). A test using this model did not reveal a significant difference in type of implant $(\mathrm{p}=0.286)$, so this variable was omitted (Figure 2). Thus, a simplified statistical model using three of the initial eight explanatory variables (length of implant, sex and presence or absence of vestibuloplasty) was found to be the best model by analysis of covariance. A test using this model revealed a greater amount of resorption in men than in women $(\mathrm{p}=0.004$; Figure 3$)$, a tendency for greater bone resorption when vestibuloplasty was performed $(\mathrm{p}=0.003$; Figure 4$)$, and more bone resorption was seen with greater implant length $(\mathrm{p}=0.002$; Figure 5$)$.

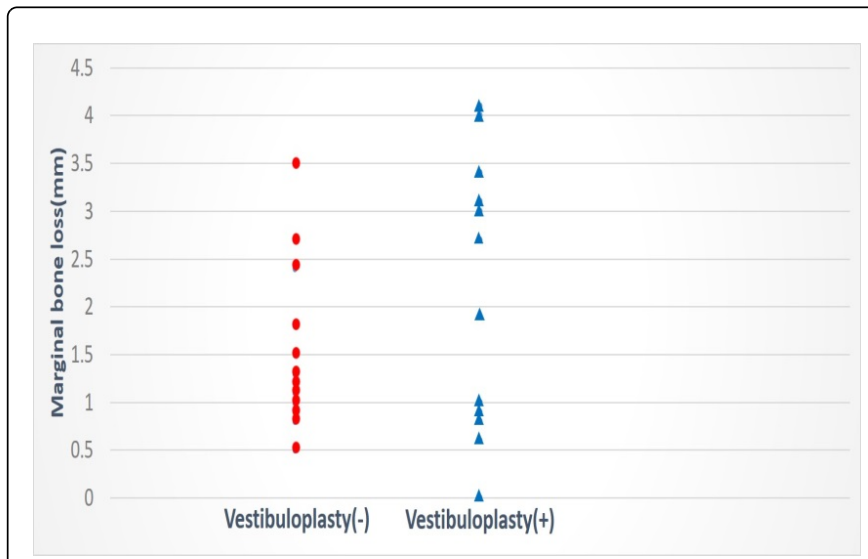

Figure 4: Effect of implant length on bone peri-implant bone loss. Amount of bone loss increased with implant length ( $\mathrm{p}=0.003)$.

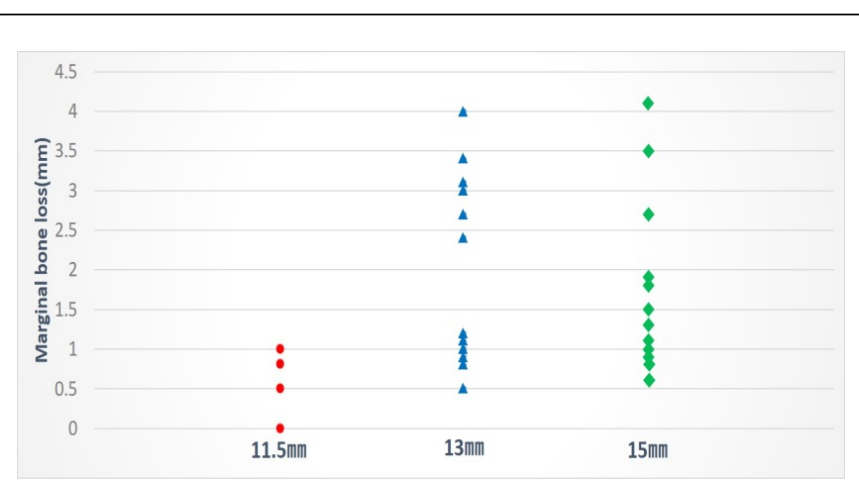

Figure 5: Effect of vestibuloplasty on peri-implant bone loss. Subjects that underwent vestibular extension exhibited a significantly greater amount of bone loss $(\mathrm{p}=0.002)$.

\section{Discussion}

Several reports have focused on the clinical outcome of implants placed in alveolar cleft sites [5-7], however, few reports have examined the effects of implants placed in alveolar cleft sites for long-term changes of peri-implant bone loss. The facts that affect the bone loss by the implant placed into alveolar cleft sites were unclear.

The surface properties of implants were previously known to affect the success of implant osseointegration. The rate success of osseointegration is reported to be significantly higher with rough surface-type implants than with machined surface-type. This was the 
case when both machined surface type titanium implants and rough surface-type titanium implants were compared, and when the bone quality of the bone or initial fixation of the surface-type implant during placement was poor [11,12]. The surface properties of almost all implants currently on the market are rough surface-type. However, peri-implantitis is prone to occurring in rough surface-type implants, and once peri-implantitis has occurred, it is difficult to control $[8,9]$. Matsui et al. analyzed changes to bone surrounding implants placed in cleft sites and reported an increasing trend in the amount of bone loss, with the smallest amount of bone loss occurring with machined surface-type implants, followed by HA-coated implants and rough surface-type implants [5]. In the present study, no statistically significant differences in the amount of bone resorption were seen by surface property in HA-coated implants, machined surface-type implants, and rough surface-type implants. One likely reason for the lack of a difference in the amount of bone loss by type of implant is that peri-implantitis was prevented by performing regular maintenance in all subjects without causing implant-related differences in oral hygiene.

There is no clear evidence that sex affects implant prognosis. Even if postmenopausal women develop osteoporosis and subsequent thinning of cortical bone and coarse trabecular bone, the activation of bone as a result of implant placement means that these issues do not hinder implant treatment if sufficient time is given for osseointegration to succeed $[13,14]$. Furthermore, the shape of the bone bridge formed by bone grafting has been found to be unaffected by sex [15]. A report that histopathologically analyzed changes following bone graft to the cleft found that approximately 6 months after bone grafting, the grafted bone had been replaced by a structure similar to alveolar bone as a result of remodeling and further confirmed that this change was not influenced by sex [16]. The mean age of the patients examined in the present study was young at 19.8 years, so female hormones were not thought to have affected bone. While it is not possible to clarify the reason for the significantly greater amount of peri-implant bone loss in men than in women in the present study based on the above, it is conceivable that changes to peri-implant bone, such as oral hygiene status and occlusion-related load applied to implants, had an effect.

The presence of attached gingiva around the implant is considered best to inhibit the onset of peri-implantitis and maintain the implant long-term $[17,18]$. In general, alveolar cleft that have undergone bone grafting have a layer of extended labial mucosa covering the grafted bone, which tends to make the oral vestibule shallower. As a result, the oral vestibule surrounding the implant placed in the cleft sites is often shallow with missing attached gingiva and movable mucosa surrounding the implant. In such cases, because peri-implantitis is prone to occurring, it is considered necessary to achieve attached gingiva surrounding the implant by means of vestibuloplasty and palatal mucosa graft. In fact, 12 subjects in the present study underwent vestibuloplasty during abutment connection. However, the amount of peri-implant bone loss was significantly greater in the group that underwent vestibuloplasty. This is likely because of surgical stress [19], which may need to be further examined in terms of surgical duration, such as performing vestibuloplasty prior to implant placement in subjects who require vestibuloplasty.

The length of the implant also affects implant prognosis. It is therefore considered best to insert implants of at least $10 \mathrm{~mm}$ in length [8]. In the present study, analysis of the length of implants and periimplant bone loss revealed a tendency for a greater amount of bone loss in subjects with longer implants, which was a finding that differed from previous reports. While the results of the present analysis do not clarify why a greater amount of bone resorption was seen in subjects with longer implants, they did suggest that the need for bone grafting around the implant during implant placement is a factor in subjects in whom long implants were chosen.

In implant treatment for alveolar cleft, a variety of factors that are not seen in non-cleft, healthy individuals, such as bone defects in the cleft area, loss of gingiva and/or mucosa, and skeletal malocclusion, are intricately linked. Detailed analysis of factors that affect peri-implant bone loss is therefore needed in a larger subject sample to establish guidelines for implant treatment of alveolar cleft.

\section{References}

1. Turvey TA, Vig K, Moriarty J, Hoke J (1984) Delayed bone grafting in the cleft maxilla and palate: a retrospective multidisciplinary analysis. Am J Orthod 86: 244-256.

2. Bergland O, Semb G, Abyholm FE (1986) Elimination of the residual alveolar cleft by secondary bone grafting and subsequent orthodontic treatment. Cleft Palate J 23: 175-205.

3. Laine J, Vähätalo K, Peltola J, Tammisalo T, Happonen RP (2002) Rehabilitation of patients with congenital unrepaired cleft palate defects using free iliac crest bone grafts and dental implants. Int J Oral Maxillofac Implants 17: 573-580.

4. Fukuda M, Takahashi T, Iino M (2003) Dentoalveolar reconstruction of a missing premaxilla using bone graft and endosteal implants. J Oral Rehabil 30: 87-90.

5. Matsui Y, Ohno K, Nishimura A, Shirota T, Kim S, et al. (2007) Longterm study of dental implants placed into alveolar cleft sites. Cleft Palate Craniofac J 44: 444-447.

6. Wang F, Wu Y, Zou D, Wang G, Kaigler D (2014) Clinical outcomes of dental implant therapy in alveolar cleft patients: a systematic review. Int J Oral Maxillofac Implants 29: 1098-1105.

7. de Barros Ferreira S Jr, Esper LA, Sbrana MC, Ribeiro IW, de Almeida AL (2010) Survival of dental implants in the cleft area--a retrospective study. Cleft Palate Craniofac J 47: 586-590.

8. Esposito M, Hirsch JM, Lekholm U, Thomsen P (1998) Biological factors contributing to failures of osseointegrated oral implants (?) Success criteria and epidemiology. Eur J Oral Sci 106: 527-551.

9. Esposito M, Hirsch JM, Lekholm U, Thomsen P (1998) Biological factors contributing to failures of osseointegrated oral implants (?) Etiopathogenesis. Eur J Oral Sci 106: 721-764.

10. Crawley MJ (2007) The R book. John Wiley \& Sons Ltd, Chichester, England.

11. Fugazzotto PA, Wheeler SL, Lindsay JA (1993) Success and failure rates of cylinder implants in type IV bone. J Periodontol 64: 1085-1087.

12. Wennerberg A, Albrektsson T, Johansson C, Andersson B (1996) Experimental study of turned and grit-blasted screw-shaped implants with special emphasis on effects of blasting material and surface topography. Biomaterials 17: 15-22.

13. Elsubeihi ES, Zarb GA (2002) Implant prosthodontics in medically challenged patients: the University of Toronto experience. J Can Dent Assoc 68: 103-108.

14. Holahan CM, Koka S, Kennel KA, Weaver AL, Assad DA, et al. (2008) Effect of osteoporotic status on the survival of titanium dental implants. Int J Oral Maxillofac Implants 23: 905-910.

15. Ames JR, Ryan DE, Maki KA (1981) The autogenous particulate cancellous bone marrow graft in alveolar clefts. A report of forty-one cases. Oral Surge Oral Med Oral Pathol 51: 588-591.

16. Iino M, Ishii H, Sato J, Seto K (2000) Histological evaluation of autogenous iliac particulate cancellous bone and marrow grafted to alveolar clefts-a preliminary report of five young adult cases. Cleft Palate Craniofac J 37: 55-60. 
Citation: Suzuki M, Kamatani T, Akizuki A, Yasuda A, Sato H, et al. (2016) Peri-Implant Bone Loss Evaluation Around Dental Implants Placed into Alveolar Cleft Sites. Dentistry 6: 398. doi:10.4172/2161-1122.1000398

Page 5 of 5

17. Simons AM, Baima RF (1994) Free gingival grafting and vestibuloplasty with endosseous implant placement: clinical report. Implant Dent 3: 235-238.

18. Deppe H, Horch HH, Kolk A (2004) Microstructured dental implants and palatal mucosal grafts in cleft patients: a retrospective analysis. J Craniomaxillofac Surg 32: 211-215.
19. Abrahamsson I, Berglundh T, Glantz PO, Lindhe J (1998) The mucosal attachment at different abutments. An experimental study in dogs. J Clin Periodontol 25: 721-727. 